\title{
Comparación de vocalizaciones de Espadarana prosoblepon en Dos Tipos de Ecosistemas usando Agrupamiento Jerárquico
}

\author{
Diana C. Duque Montoya ${ }^{1}$, Claudia V. Isaza Narváez ${ }^{1}$, Estefany Cano Rojas ${ }^{2}$ \\ diana.duquem@udea.edu.co, victoria.isaza@udea.edu.co, canoestefany@gmail.com \\ ${ }^{1}$ SISTEMIC - Universidad de Antioquia, calle 70 No. 52 - 21, AA 1226, Medellín, Colombia. \\ ${ }^{2}$ GHA - Universidad de Antioquia, calle 70 No. 52 - 21, AA 1226, Medellín, Colombia.
}

DOI: 10.17013/risti.24.13-21

\begin{abstract}
Resumen: Se analizaron las vocalizaciones de la Espadarana prosoblepon en dos ecosistemas diferentes, un bosque conservado cerca de una represa en el suroeste de Antioquia - Colombia y un bosque alterado en Carmen de Atrato, Chocó - Colombia Las grabaciones fueron segmentadas, luego procesadas para extraer y seleccionar los descriptores y finalmente clasificadas usando un algoritmo jerárquico. Con el fin de generalizar el análisis, también se procesaron las vocalizaciones de la Sachatamia albomaculata. Los resultados muestran que las vocalizaciones de la misma especie son afectadas por el ambiente, y que los tres conjuntos de vocalizaciones pueden ser diferenciados con la metodología propuesta.
\end{abstract}

Palabras clave: Espadarana prosoblepon, bioacústica, dendrograma, ecosistemas.

Comparison of Espadarana Prosoblepon Vocalizations in Two Different Ecosystems using Hierarchical Chstering

\begin{abstract}
Espadarana prosoblepon vocalizations were analyzed in two different ecosystems, a conserved forest near to a dam in southeast Antioquia and a disturbed forest in Carmen de Atrato, Chocó. The recordings were segmented, then processed to extract and select features and classified with a hierarchical algorithm. To analyze more generally the results, Sachatamia albomaculata vocalizations from the dam were processed too. The results show that the species vocalizations are clearly affected by the environment, and the three types of vocalizations can be separated with the proposed methodology.
\end{abstract}

Keywords: Espadarana prosoblepon, bioacoustics, dendrogram, ecosystems.

\section{Introducción}

La bioacústica es una ciencia que estudia el comportamiento de las especies mediante los sonidos que estas producen (Fletcher, 2007). Los beneficios de esta ciencia son la 
posibilidad de estudiar especies muy raras que no son fáciles de encontrar en salidas de campo; el incremento del tiempo de estudio de las señales biológicas, por la capacidad de las grabadoras de funcionar por largos periodos de tiempo; y la poca afectación al ecosistema que causa al compararse con la recolección de ejemplares de las especies.

Actualmente, el GHA (Grupo Herpetológico de Antioquia) de la Universidad de Antioquia, trabaja en el monitoreo de anuros basados en sus vocalizaciones en el área que rodea la hidroeléctrica Jaguas, de Isagen (Bedoya, Isaza, Daza, \& López, 2014; Daza, 2013). Los anuros son considerados una especie indicadora, porque son muy sensibles a los cambios en el ecosistema, por lo que pueden indicar la degradación del hábitat (Bedoya, 2015). Además, sus vocalizaciones tienen la ventaja de ser, en gran mayoría, monosilábicas y los cantos de la misma especie se encuentran por lo general, en la misma banda de frecuencia.

El objetivo de este estudio es analizar las diferencias en el espectrograma de los cantos de dos poblaciones de la misma especie de anuros, pero localizadas en diferentes lugares: Jaguas, una represa en el sureste de Antioquia, en Colombia y Carmen de Atrato, un pequeño pueblo del Chocó, también en Colombia. El primer hábitat es un bosque conservado, cuya única alteración es la represa construida cerca de este. El otro hábitat es un bosque intervenido por el ser humano. Para comparar los resultados y evaluar los cambios de los cantos de la especie, también se consideraron los cantos de otra especie de la misma familia. La metodología se basa en la descomposición en valores singulares de los descriptores extraídos, y en técnicas de agrupamiento jerárquico para obtener diferente cantidad de grupos por nivel de similitud.

\section{Materiales y Métodos}

\subsection{Materiales}

La especie escogida para este estudio es la Espadarana prosoblepon, un anuro de la familia Centrolenidae. Este anuro habita centro y suramérica, desde Honduras hasta Ecuador; es una especie nocturna y sus cantos tienen una frecuencia dominante alrededor de los $5 \mathrm{KHz}$ (Richman, 2008). Las vocalizaciones de la Sachatamia albomaculata fueron usadas para comparar los resultados obtenidos, esta especie pertenece a la misma familia Centrolenidae pero la banda de frecuencia de sus cantos está alrededor de los $6 \mathrm{KHz}$ (Chantasirivisal, 2005). Las vocalizaciones de esta segunda especie se obtuvieron de las grabaciones de Jaguas únicamente.

Para este estudio se procesaron 58 grabaciones con cantos de las dos especies, 28 grabaciones de Jaguas, que fueron registradas en Noviembre del 2012 y 30 grabaciones de Carmen de Atrato, registradas en Octubre del 2015. Las señales se obtuvieron con micrófonos omnidireccionales usando una grabadora Song Meter SM2. Esta grabadora fue programada para grabar durante un minuto, con frecuencia de muestreo de $44100 \mathrm{~Hz}$ y guardar las grabaciones en formato WAC, que posteriormente fueron transformadas sin pérdida a formato WAV.

\subsection{Métodos}

En la propuesta realizada para el análisis de las grabaciones, primero se hace una segmentación de las vocalizaciones, es decir, la identificación y extracción de las partes de 
las grabaciones con cantos de las ranas; estos segmentos fueron caracterizados en tiempo y frecuencia. El segundo paso es la extracción de características; dado que el sonido es solo una señal, se calcularon los coeficientes cepstrales de frecuencia para describir cada segmento usando diferentes variables; luego se transformaron estas variables para reducir su número mediante una técnica de filtrado basada en la descomposición en valores singulares; y por último se clasificaron los datos.

\section{Segmentación}

La segmentación se hizo de forma manual. Para llevarla a cabo, se inspeccionaron visualmente los espectrogramas de las señales y cuando un canto era reconocido, la banda de frecuencia y la ventana de tiempo se registraban para ser ingresados al extractor de características. En la figura 1, se presenta un ejemplo de los segmentos espectrales de ambas especies en los dos hábitats estudiados.

\section{Extracción de Características}

Para la extracción automática de características, se usó una versión modificada de los coeficientes cepstrales de Mel (MFCC). Los MFCC son muy utilizados para el reconocimiento automático del habla (Mermelstein, 1976). Sin embargo, esta técnica se usa para la identificación de vocalizaciones humanas, ya que se aplican los filtros de frecuencia de Mel que extraen coeficientes según el sistema auditivo humano, por lo que no es recomendable para las vocalizaciones de otras especies. Por lo tanto, el uso de estos filtros fue omitido durante el proceso, y de esta manera obtener coeficientes uniformemente y linealmente distribuidos a lo largo del espectro (Bedoya, 2015). El procedimiento fue el siguiente:

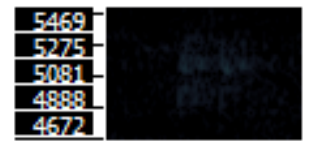

(a)

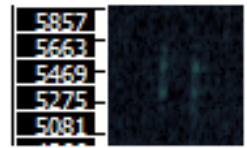

(b)

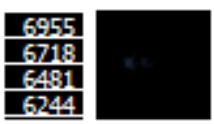

(c)

Figura 1 - Segmentos espectrales de los cantos de las ranas. A la derecha de cada segmento se muestra la escala de frecuencia en Hz. (a) Canto de la Espadarana prosoblepon en Jaguas. (b) Canto de la Espadarana prosoblepon en Carmen de Atrato. (c) Canto de la Sachatamia albomaculata en Jaguas.

1. Se divide cada segmento en partes.

2. Se calcula la potencia espectral de cada parte usando la transformada rápida de Fourier (FFT) 
3. Se obtiene el logaritmo de la energía de todas las partes del segmento.

4. Por último, se estima la transformada de coseno discreta de los resultados para obtener los coeficientes cepstrales.

El número de coeficientes es determinado por el tipo de aplicación. Para este caso se usaron 100 coeficientes, basándose en un trabajo previo (Bedoya, 2015).

\section{Selección de Características}

De la etapa anterior se obtuvieron 100 variables por canto, lo que dificulta su procesamiento y visualización; por lo tanto, se optó por un método estadístico para reducir la dimensionalidad del problema. El escalamiento multidimensional (EMD) es un método basado en la descomposición en valores singulares (Golub \& Kahan, 1965) para transformar las variables originales en un nuevo conjunto de variables que conserve la distancia entre las muestras (Kruskal, 1964). Se aplicó este método porque la técnica de agrupamiento a utilizar se basa en distancia, por lo que se esperan resultados aproximados a los que se habrían obtenido con el uso de las variables originales.

Este método comienza con el cálculo de la matriz de distancia euclidiana estandarizada $\mathbf{D}_{\mathrm{nxn}}$, donde $\mathrm{n}$ es el número de muestras y $\delta_{\mathrm{ij}}$ representa a cada uno de sus elementos. Luego se obtienen las matrices A y $\mathbf{B}$ usando (1) y (2) respectivamente; a continuación, se extrae la matriz $\mathbf{Z}$ usando descomposición en valores singulares para obtener las nuevas variables como columnas de la nueva matriz (ver (3)). Los valores propios de la matriz $\mathbf{B}$ representan la significancia de las nuevas variables, es decir, las variables con los valores propios más altos son las más significativas para representar la muestra.

$$
\begin{gathered}
\boldsymbol{A}_{n \times n}=\left(a_{i j}\right)=\left(-\frac{1}{2} \delta_{i j}^{2}\right) \\
\boldsymbol{B}_{n \times n}=\left(b_{i j}\right)=\left(\boldsymbol{I}-\frac{1}{n} \boldsymbol{J}\right) \boldsymbol{A}\left(\boldsymbol{I}-\frac{1}{n} \boldsymbol{J}\right) \\
\boldsymbol{B}=\boldsymbol{V} \boldsymbol{\Lambda} \boldsymbol{V}^{T}=\boldsymbol{V} \boldsymbol{\Lambda}^{\frac{1}{2}} \boldsymbol{\Lambda}^{\frac{1}{2}} \boldsymbol{V}^{T}=\boldsymbol{Z} \boldsymbol{Z}^{T}
\end{gathered}
$$

En (2) I es la matriz de identidad nxn, J es una matriz nxn en donde todos sus elementos son uno; en (3) $\mathbf{V}$ y $\mathbf{\Lambda}$ son las matrices resultantes de la descomposición en valores singulares, y los elementos de la diagonal de $\boldsymbol{\Lambda}$ son las raíces cuadradas de los valores propios de $\mathbf{B}$ o el stress de las nuevas variables.

\section{Agrupamiento}

Se eligió el agrupamiento jerárquico para clasificar los datos (Jiawei \& Kamber, 2001). De esta manera se dejan en un mismo grupo los cantos que se consideran comunes. Las variables más significativas obtenidas del paso anterior se usaron para calcular la distancia entre muestras, luego estas son agrupadas por su proximidad; se inicia con 
grupos de un solo elemento hasta agrupar todos los elementos en un solo grupo. Lo que es interesante es analizar las clases que se forman durante este proceso para estudiar los diferentes niveles de similitud entre las muestras. La medida de similitud fue la distancia euclidiana estandarizada, como en el paso anterior, y las muestras fueron conectadas usando enlace simple (entre vecinos más cercanos) y enlace promedio para comparar.

\section{Resultados}

De la etapa de segmentación, 91 cantos de la Espadarana prosoblepon y 54 cantos de la Sachatamia albomaculata fueron encontrados en las grabaciones de Jaguas; y 63 cantos de la Espadarana prosoblepon fueron encontrados en las grabaciones de Carmen de Atrato. Los cantos de la Espadarana prosoblepon estaban todos en la misma banda de frecuencia, aunque los cantos de Carmen de Atrato fueron más difíciles de detectar debido al ruido presente en las grabaciones, al parecer porque la grabadora se encontraba cerca de un arroyo y la lluvia era frecuente. Fue interesante notar que las vocalizaciones de las ranas de Jaguas se encontraron en la franja horaria entre la 1 y las 5 de la mañana, mientras que las de Carmen de Atrato se encontraron entre las 6 y 10 de la noche.

Luego de la etapa de extracción de características, el escalamiento multidimensional fue ejecutado. El stress de todos los valores propios encontrados se muestra en la figura 2.

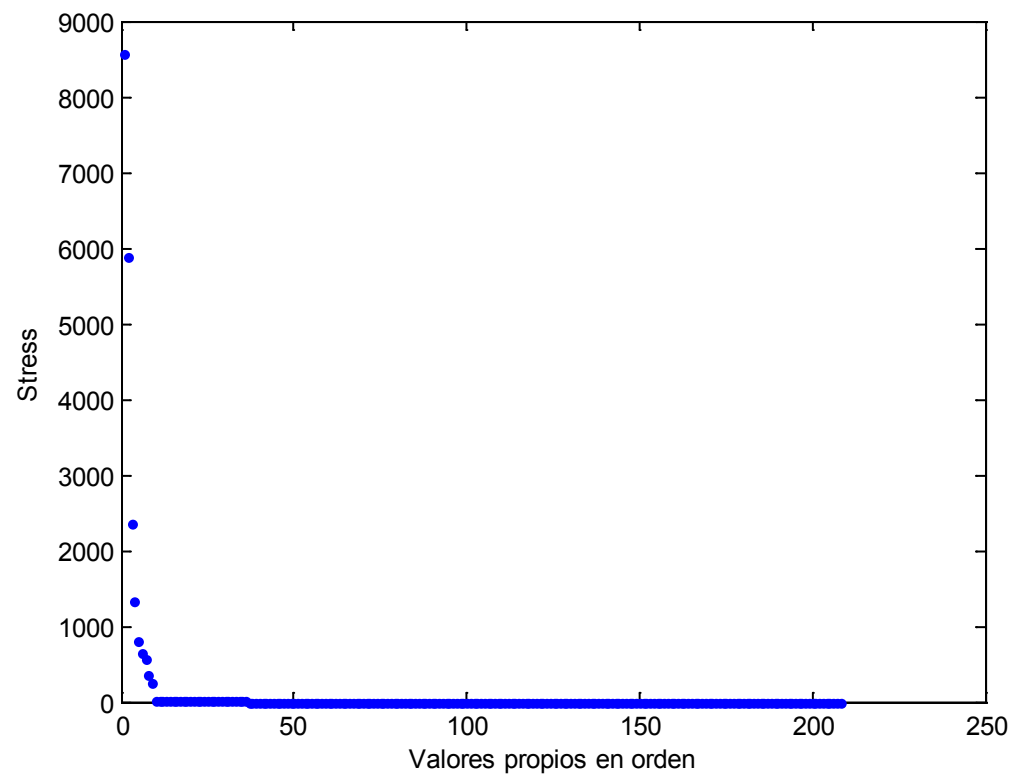

Figura 2 - Stress obtenido del escalamiento multidimensional.

La figura 2 muestra que las primeras nueve variables son las más significativas, lo que quiere decir que al calcular la distancia entre estas nueve nuevas variables es casi lo 
mismo que calcular la distancia con las 100 variables originales. Estos fueron entonces los descriptores escogidos para realizar el agrupamiento.

En la figura 3 se grafica la variable 1 versus la variable 2 para todas las muestras. Los puntos azules son las vocalizaciones de la Espadarana prosoblepon de Carmen de Atrato, los puntos rojos son las vocalizaciones de la misma especie en Jaguas, y los puntos verdes son las vocalizaciones de la Sachatamia albomaculata. Es fácil identificar que las dos poblaciones de la misma especie se pueden separar, lo que quiere decir que las vocalizaciones de Jaguas son diferentes de las vocalizaciones del Chocó para la misma especie. Sin embargo, la diferencia con las vocalizaciones de la otra especie no es tan grande como se esperaba, puede ser incluso posible que la clase de la Espadarana prosoblepon del Carmen de Atrato se agrupe primero con la clase de la Sachatamia albomaculata que con la clase de la Espadarana prosoblepon de Jaguas; esto se describe más adelante. Como comentario adicional, las varianzas de las vocalizaciones de Jaguas parecen ser mayores a la de Carmen de Atrato, debido probablemente a que las ranas de Jaguas tienen una banda de frecuencias más amplia para cantar, mientras que las ranas en Carmen de Atrato estaban más limitadas por el ruido, por lo que el nicho acústico era más reducido.

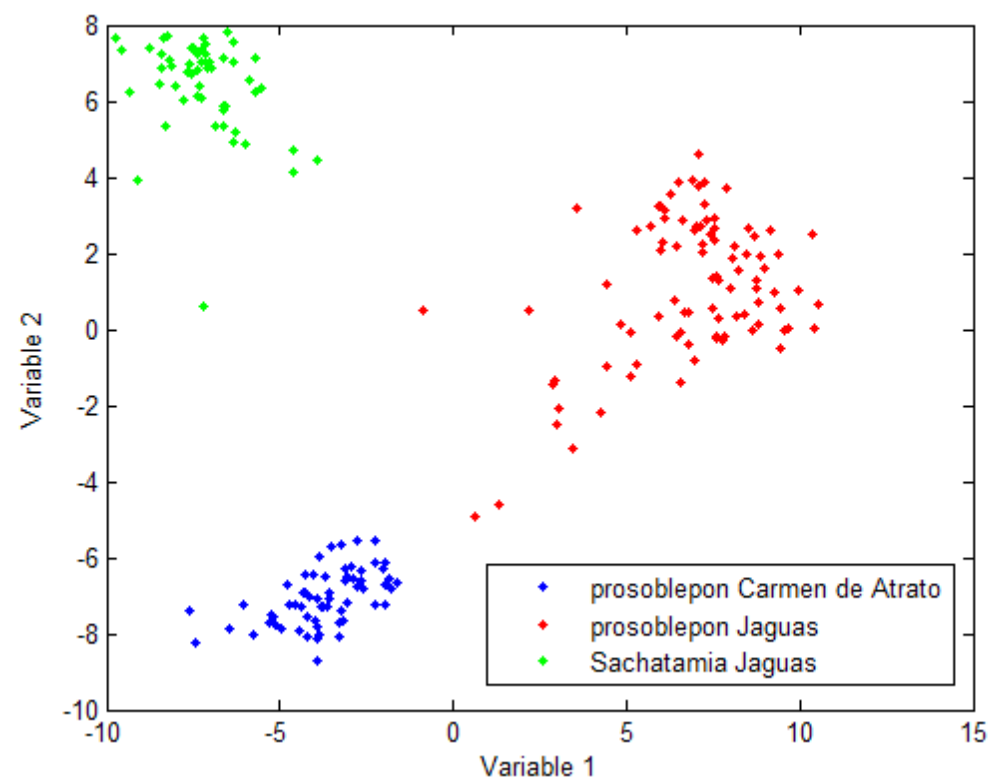

Figura 3 - Las muestras son graficadas en un espacio de dos dimensiones formado por la variable 1 y la variable 2 obtenidas del EMD. Los puntos azules son las vocalizaciones de la prosoblepon de Carmen de Atrato, los puntos rojos son las vocalizaciones de la prosoblepon de Jaguas y los puntos verdes son vocalizaciones de la Sachatamia.

Finalmente, se utiliza el agrupamiento jerárquico con el propósito d e encontrar las similitudes dentro y entre especies. El dendrograma de enlace simple se muestra en la figura 4 . 


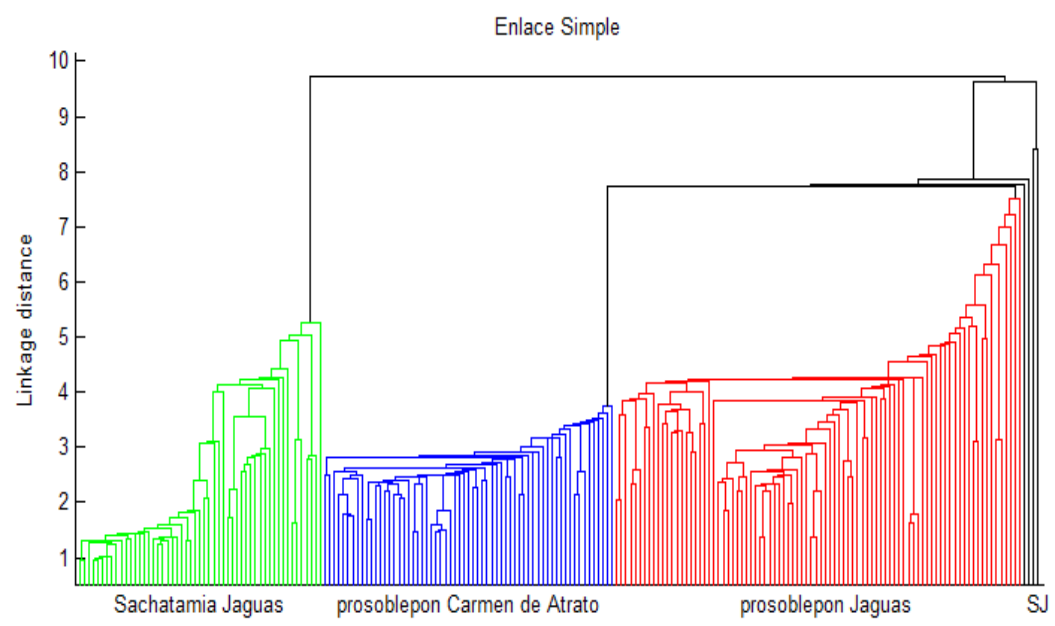

Figura 4 - Dendrograma de enlace simple de los datos. Los colores son los grupos encontrados automáticamente correspondientes a las tres poblaciones de ranas, las ramas negras son datos atípicos.

Las muestras de la Espadarana prosoblepon en Carmen de Atrato coincidieron con las ramas azules, las muestras de la misma especie de Jaguas coincidieron con las ramas rojas (excepto por tres muestras atípicas), y las muestras de la Sachatamia albomaculata coincidieron con las ramas verdes, excepto por un dato atípico. Las diferencias en las varianzas de los grupos explican la razón por la que las vocalizaciones de Carmen de Atrato son agrupadas primero en el dendrograma. Para un monitoreo biológico pude ser interesante analizar los subgrupos de cada clase para extraer información de esos individuos, especialmente para las muestras de Jaguas. También se pueden ver los datos atípicos como ramas negras, los cuales representan 4 errores del algoritmo al compararse con las etiquetas originales, el error fue entonces del $1.92 \%$

También se implementó un dendrograma de enlace promedio para disminuir el efecto de los datos atípicos. Este se muestra en la figura 5.

En este caso, las muestras de Sachatamia y Carmen de Atrato se agrupan en una clase antes de agruparse con las muestras de la prosoblepon de Jaguas. Esto demuestra que la influencia de los factores ambientales puede causar mayor similitud entre vocalizaciones de diferente especie que entre vocalizaciones de la misma especie, pero en hábitat diferente. Comparando con las etiquetas originales, obtenidas por un análisis individual realizado por herpetólogos, el clasificador propuesto solamente tuvo dos errores, una muestra de Sachatamia y una de la prosoblepon de Jaguas clasificadas ambas como prosoblepon de Carmen de Atrato lo que corresponde a un error del $0.96 \%$. 


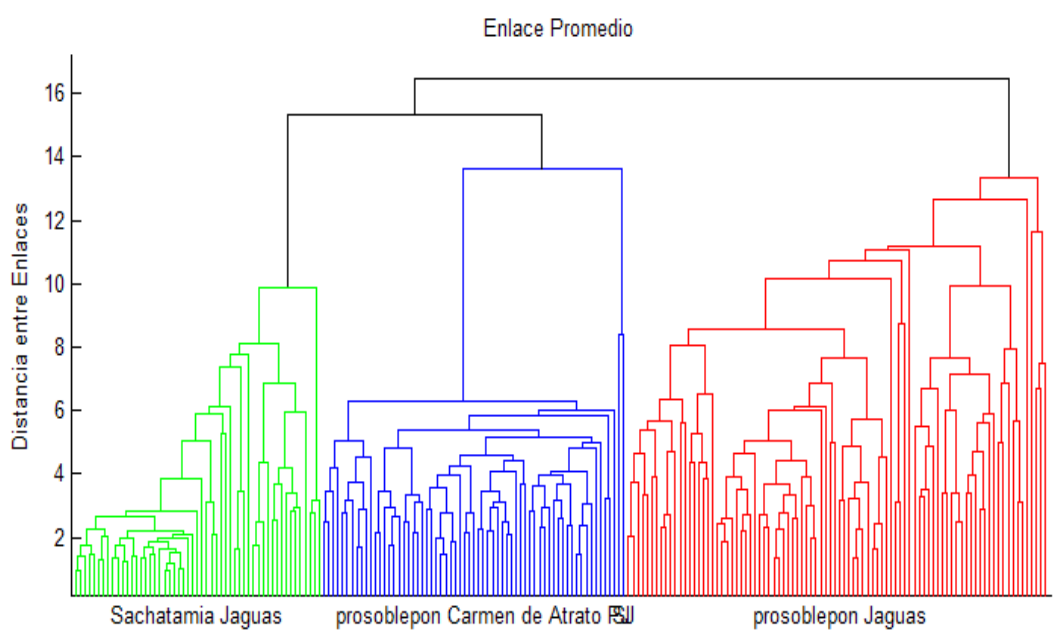

Figura 5 - Dendrograma de enlace promedio de los datos. Los colores son los grupos encontrados automáticamente correspondientes a las tres poblaciones de ranas.

\section{Conclusiones}

La metodología utilizada fue apropiada para el problema, ya que los grupos pudieron ser fácilmente separados usando distancia euclidiana estandarizada como medida de similitud; además, el escalamiento multidimensional ayudó a reducir el número de variables con poca pérdida de información de los datos originales.

Este estudio prueba que el monitoreo acústico puede detectar la influencia que el ambiente causa en las vocalizaciones de una misma especie. Para un estudio relacionado con el monitoreo bioacústico, con la propuesta realizada se pueden percibir cambios en el comportamiento de los anuros y analizar los subgrupos más representativos para estudiar las características fonéticas de las especies. Es interesante notar que en ninguno de los dos dendrogramas las muestras de Jaguas se agruparon primero que las de Carmen de Atrato; este hecho refuerza la hipótesis del nicho acústico, que supone que las especies diferentes dentro de un mismo lugar intentan diferenciar sus vocalizaciones para mejorar la comunicación entre los individuos de la misma especie (Krause, 1993).

\section{Agradecimientos}

Gracias al Grupo Herpetológico de Antioquia por el suministro de la base de datos y a Isagen por permitir el monitoreo de los ecosistemas alrededor de la hidroeléctrica Jaguas.

\section{Referencias}

Bedoya, C., Isaza, C., Daza, J. M., \& López, J. D. (2014). Automatic recognition of anuran species based on syllable identification. Ecological Informatics, 24, 200-209. http://doi.org/10.1016/j.ecoinf.2014.08.009. 
Bedoya, C. L. (2015). Unsupervised Identification of Anuran Vocalizations. (Master's thesis). Medellín, Colômbia: Universidad de Antioquia.

Chantasirivisal, P. (2005). AmphibiaWeb - Sachatamia albomaculata. Retrieved from http://amphibiaweb.org/cgi/amphib_query?where-genus=Sachatamia\&wherespecies=albomaculata.

Daza, J. M. (2013). Monitoreo de algunas poblaciones de vertebrados endémicos y amenazados presentes en las Centrales Hidroeléctricas Jaguas y San Carlos en el Oriente Antioqueño (Rep. No. 46/4208). Medellín: Universidad de Antioquia.

Fletcher, N. (2007). Animal Bioacoustics. In T. D. Rossing (Ed.), Springer Handbook of Acoustics (1st ed.), pp. 473-491. New York: Springer New York. http://doi. org/10.1007/978-0-387-30425-O_19.

Golub, G., \& Kahan, W. (1965). Calculating the Singular Values and Pseudo-Inverse of a Matrix. Journal of the Society for Industrial and Applied Mathematics Series B Numerical Analysis, 2, 205-224. http://doi.org/10.1137/0702016.

Han, J., Kamber, M., \& Pei, J. (2012). Data mining: concepts and techniques ( $3^{\text {rd }}$ ed). Waltham, MA: Elsevier.

Krause, B. L. (1993). The Niche Hypothesis: A Virtual Symphony of Animal Sounds, The Origins of Musical Expression and the Health of Habitats. The Soundscape Newsletter, 6, 6-10.

Kruskal, J. B. (1964). Multidimensional scaling by optimizing goodness of fit to a nonmetric hypothesis. Psychometrika, 29(1), 1-27. http://doi.org/10.1007/ BFo2289565.

Mermelstein, P. (1976). Distance measures for speech recognition, psychological and instrumental. Pattern Recognition and Artificial Intelligence, 116, 374-388.

Richman, S. (2008). AmphibiaWeb - Espadarana Prosoblepon. Retrieved from http://amphibiaweb.org/species/1741. 